\title{
IDENTIFICACIÓN DE RAZAS DEL NEMATODO DE QUISTE DE PAPA (Globodera pallida (Stone)) CON DIFERENCIALES O CLONES DE PAPA
}

\author{
Ricardo Piedra Naranjo ${ }^{1}$, Miguel Obregón Gómez², Cristina Vargas Chacón ${ }^{3}$, \\ Jeannette Avilés Chaves ${ }^{4}$, Jorge Meckbel Campos ${ }^{5}$
}

\begin{abstract}
RESUMEN
El estudio se realizó en la localidad de San Juan de Chicuá, provincia de Cartago, a 5 kilómetros del Volcán Irazú, con una altura de 2.800 metros sobre el nivel del mar y a 26 kilómetros al noreste de la provincia de Cartago. Utilizando diferenciales o clones de papa se ejecutó la investigación para determinar razas del nematodo Globodera pallida (Stone) en el cultivo. La identificación de los patotipos se hizo basado en la tasa de reproducción de las distintas poblaciones en una serie standard de clones de Solanum spp. Con una viabilidad de 260 huevos o larvas por quiste, fue inoculado cada diferencial, con un número de 35, 40 y 45 quistes por pote. Se tomó temperatura y humedad de suelo. Los clones de papa o diferenciales utilizados fueron: Floresta (Solanum tuberosum), 800286 (Multidissectum híbrido P55/ 7( $\mathrm{H}_{2}$ ), 800289 (KTT, 60.21.19), 800290 (GLK 58.1642.4), 800291(VTn 62.33.3), 800944 (65.346.19). Se observó que los clones positivos presentaron reacciones importantes a la raza P3A de Globodera pallida Stone. Los diferenciales o clones 800289, 800290 y 800291 mostraron resistencia a la plaga. Las razas de esta plaga pueden cambiar por evolución de la misma, al someterse a otras variedades o nuevos huéspedes, sin embargo, el estudio es importante para crear un programa con variedades tolerantes o resistentes y esto contribuye sin duda dentro de elementos para un manejo integrado de la plaga dentro de las buenas prácticas agronómicas del cultivo de papa en Costa Rica.
\end{abstract}

Palabras claves: Biotipo o raza, diferenciales o clones de papa, resistencia o tolerancia

\footnotetext{
${ }^{1}$ Instituto Nacional de Innovación y Transferencia en Tecnología Agropecuaria. (506)2231-5055. INTA. Costa Rica. Estudiante Doctorado en Ciencias Naturales Para el Desarrollo (DOCINAE).

2 Profesor de Doctorado en Ciencia Naturales Para el Desarrollo (DOCINAE). La Aurora de Heredia. Costa Rica (506)88286382.

${ }^{3}$ Instituto Nacional de Innovación y Transferencia en Tecnología Agropecuaria. (506)2231-5055. INTA. Costa Rica

${ }^{4}$ Instituto Nacional de Innovación y Transferencia en Tecnología Agropecuaria. (506)2250-1224. INTA. Costa Rica.

${ }^{5}$ Instituto Nacional de Innovación y Transferencia en Tecnología Agropecuaria. (506)2231-5055. INTA. Costa Rica.
} 


\section{INTRODUCCIÓN}

En Costa Rica, se siembran aproximadamente 3.000 hectáreas de papa, ubicadas principalmente en la zona norte de Cartago y Zarcero. De este cultivo dependen aproximadamente 1.200 agricultores. La papa en Costa Rica ocupa un papel preponderante en la canasta básica, la mayoría se consume en forma fresca, aunque en los últimos años ha aumentado la necesidad de producir papa para la industria. La principal zona productora de papa en Costa Rica se encuentra en la provincia de Cartago, donde se cultivan alrededor de 2.800 hectáreas, seguida por Zarcero que dedica a este cultivo cerca de 300 hectáreas. El rendimiento varía de 12 a 25 toneladas por hectárea. La demanda interna es mayor a 5.000 toneladas mensuales, oscilando entre 60.000 y 70.000 toneladas anuales. La mayor parte de la producción se destina a consumo en fresco, y solamente un $15 \%$ se destina a uso industrial, principalmente para la producción de hojuelas y papas fritas (Alfaro 2009).

Al igual que otros cultivos, la papa no escapa al ataque de plagas y enfermedades. El manejo de estos inconvenientes hace que en los sistemas agrícolas, sea uno de los problemas prioritarios por resolver para una producción competitiva. Esto debido a que la papa es uno de los principales cultivos en Costa Rica, es de vital importancia proveer a los productores de manejo adecuado en cuanto a las principales enfermedades y plagas del cultivo (Brenes et al. 2002). Dentro de esas limitantes de las plagas y enfermedades, el nematodo de quiste de la papa Globodera pallida spp, constituye uno de los problemas más importantes del cultivo en el mundo. Afecta el rendimiento, eleva los costos de producción y en consecuencia ocasiona escasez del tubérculo que tiene vital importancia en la alimentación humana (Franco et al.1993).

Las reducciones de rendimiento dependen del nivel poblacional del nematodo al momento de la siembra. Ensayos realizados en Europa y Chile han determinado que el límite de tolerancia de la papa a los nematodos formadores de quistes es de aproximadamente 1,90 huevos/g de suelo. El rendimiento de la papa puede ser reducido entre 20 y $50 \%$ cuando el nivel poblacional del nematodo en el suelo alcanza 16 y 32 huevos/g de suelo, respectivamente. El cultivo puede ser destruido completamente cuando la población inicial del nematodo es de 64 huevos/g de suelo. La magnitud del daño ocasionado por estos patógenos también depende del patotipo. A nivel mundial han sido identificados cinco patotipos de G. rostochiensis (Ro1, Ro2, Ro3, Ro4, Ro5), y seis de G. pallida: tres en Europa (PAa1, PAa2, PAa3) y tres en la zona andina (PA4A, PA5A, PA6A). La identificación de los patotipos se hace basándose en la tasa de reproducción de las distintas poblaciones en una serie Standard de clones de Solanum spp. (Schots1987). Métodos basados en separaciones de proteínas, enzimas y pruebas de ADN, hasta ahora, no han dado resultados satisfactorios. (Crozzoli 1993).

En la formación de quistes, aun cuando la coloración amarilla de las hembras indica claramente la presencia de G. rostochiensis, la ausencia de hembras con esta coloración en las raíces no garantiza que se trate de $G$. pallida, a menos que se observe el desarrollo del nematodo a lo largo de su ciclo biológico (Greco et al 1982). Una técnica utilizada y que tiene validez ha sido la preparación de los cortes perineales de los quistes, colectados en las raíces de la planta de papa, y el conteo de las estrías cuniculares presentes entre el ano y la vulva, constituyen una manera simple de diferenciar las dos especies. G. rostochiensis posee un promedio de 21,60 estrías y $G$. pallida 12,00 (Greco 1993).

Las variedades de papa resistentes al nematodo de quiste de la papa, son una buena alternativa para controlar esta plaga, dado que impiden que el nematodo se reproduzca en las raíces de la papa. El uso de variedades con tolerancia o resistencia no significa costos adicionales para el agricultor y evitan o reducen el uso de nematicidas, los cuales influyen negativamente sobre el ambiente y el ser humano (Huijsman 1960). 
Como parte inicial e importante de conocer esta plaga en Costa Rica; es por tal razón, que se ejecutó la siguiente investigación para determinar mediante clones o diferenciales de papa, la raza o razas del nematodo Globodera pallida Stone. Se evaluó peso en gramos de los tubérculos y la cantidad de quistes en raíz en cada diferencial inoculado. Se utilizó, para identificar las razas, una tabla de esquema latino para Globodera pallida (Stone).

\section{MATERIALES Y METODOS}

El estudio se desarrolló en San Juan de Chicuá de Oreamuno, provincia de Cartago, con una altitud de $2.800 \mathrm{msnm}$, predomina el bosque muy húmedo montano, el suelo es de origen volcánico del orden Andisol, la temperatura, precipitación y humedad relativa promedio anual es de $15 \stackrel{\circ}{\circ}, 2.100 \mathrm{~mm}$ y 85 $\%$, respectivamente.

Para la investigación en los potes o macetas sembrados por los clones, se tomó los datos de promedio de temperatura mínima y máxima (Figura 1). Lo anterior fue importante para el desarrollo de la plaga. Se utilizaron potes con medidas de $10 \times 10 \mathrm{~cm}$. El suelo para la investigación fue arenoso y esterilizado con buenas condiciones tanto para la planta y el desarrollo del ciclo del nematodo Globodera pallida (Stone).
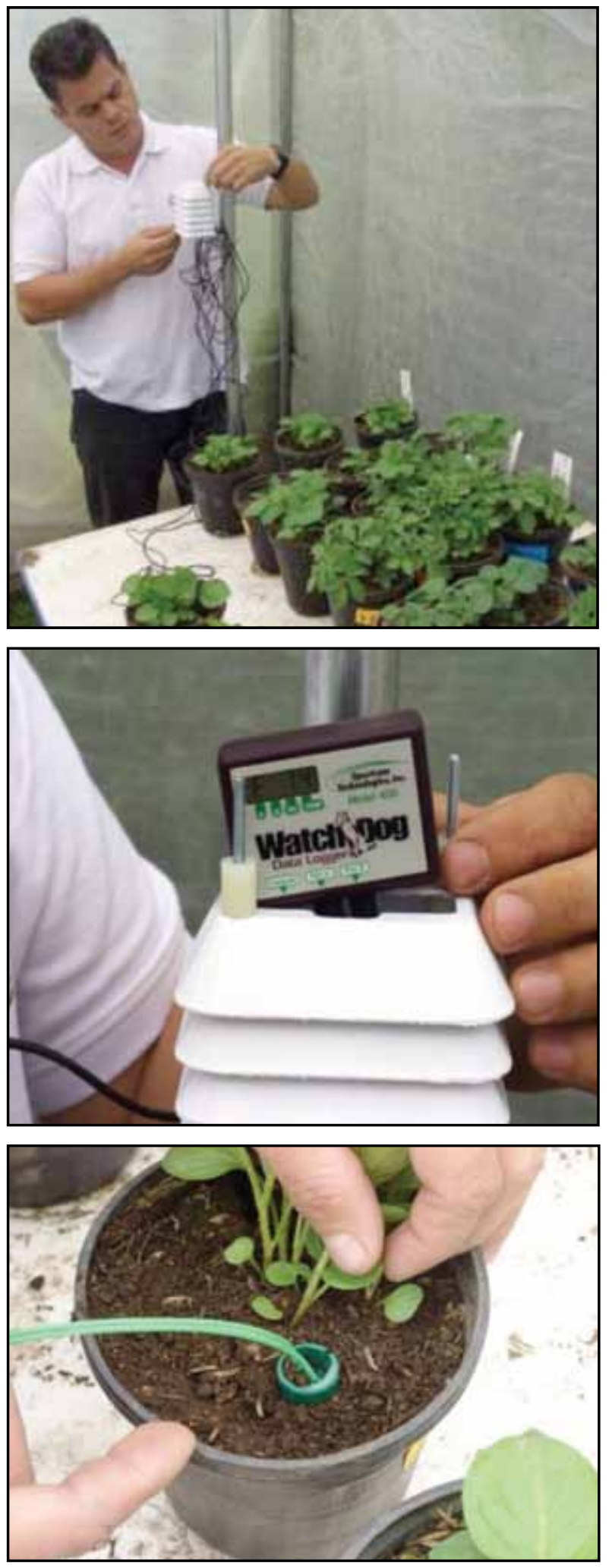

Figura 1. Ubicación de estación meteorológica para la toma de temperatura de suelo. San Juan de Chicúa, Cartago. Costa Rica. 2008. 
Se usaron potes con medidas de $10 \times 10 \mathrm{~cm}$. Se obtuvo un estricto control en la manipulación del ensayo para evitar la contaminación en la finca. Se utilizó suelo arenoso y esterilizado para un mejor desarrollo de la planta y el ciclo del nematodo Globodera pallida (Stone). De los quistes seleccionados se identificó Globodera pallida (Stone) mediante corte perineal (Figura 2).
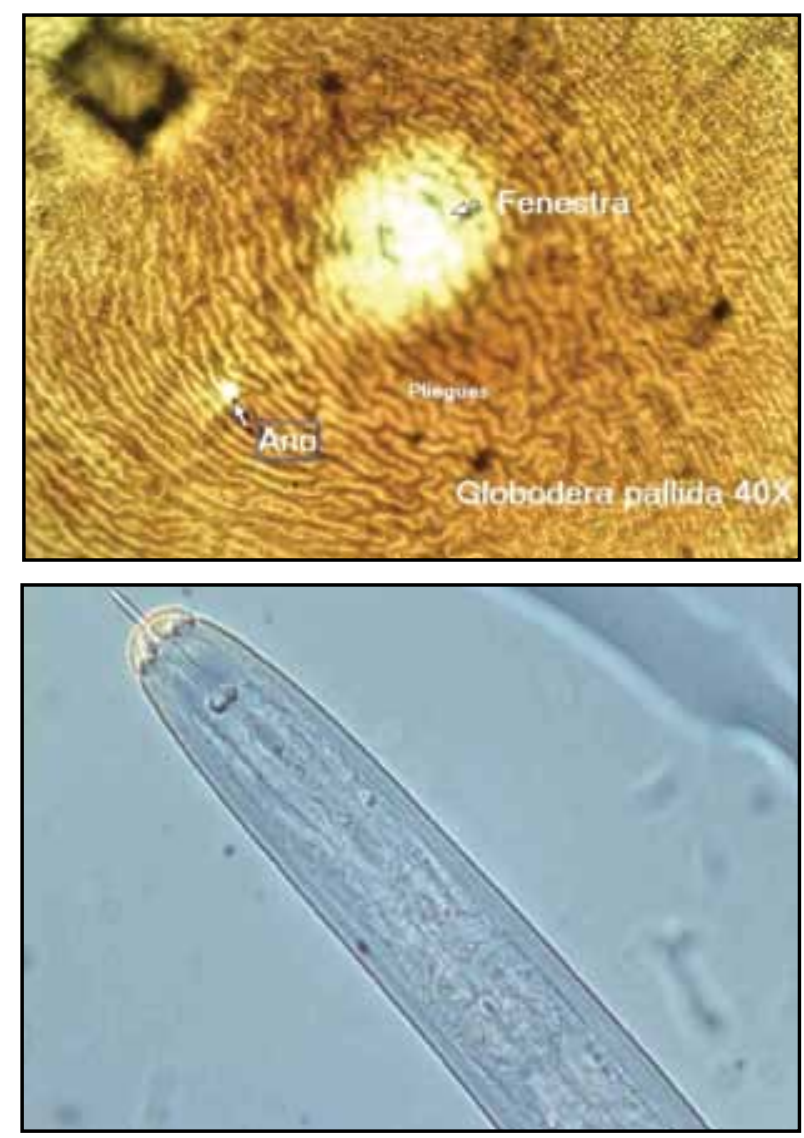

Figura 2. Identificación de Globodera pallida Stone mediante corte perineal. Fuente. Piedra Naranjo, R. 2008.

Se obtuvo quistes de $G$. pallida para la inoculación en forma homogénea (características en tamaño y color parecidas) de manera que se utilizó cada quiste una cantidad de huevos uniforme en cada tratamiento. La variedad de papa de referencia susceptible fue Floresta (Solanum tuberosum ssp tuberosum). Tanto Floresta como a los demás diferenciales se les inoculó la cantidad de 40,45 y 50 quistes (Cuadro 1).
Cuadro 1. Clones o diferenciales de papa inoculados con diferentes cantidades de quistes. San Juan de Chicuá. Cartago. 2008

\begin{tabular}{lccc}
\hline Diferenciales & $\begin{array}{c}\text { No. } \\
\text { Quistes }\end{array}$ & $\begin{array}{c}\text { No. } \\
\text { Quistes }\end{array}$ & $\begin{array}{c}\text { No. } \\
\text { Quistes }\end{array}$ \\
\hline $\begin{array}{l}\text { Floresta } \\
\text { (Solanum } \\
\text { tuberosum ssp } \\
\text { tuberosum) }\end{array}$ & 40 & 45 & 50 \\
\hline $\begin{array}{l}800286 \\
\text { (Multidissectum } \\
\text { híbrido P55/7) } \\
\text { (H2) }\end{array}$ & 40 & 45 & 50 \\
\hline $\begin{array}{l}\text { 800289 } \\
\text { (KTT 60.21.19) }\end{array}$ & 40 & 45 & 50 \\
\hline $\begin{array}{l}800290 \\
\text { (GLKS 58. }\end{array}$ & 40 & 45 & 50 \\
\begin{tabular}{l}
$1642.4)$ \\
\hline $\begin{array}{l}\text { 800291 } \\
\text { (VTn 62.33.3) }\end{array}$
\end{tabular} & & & \\
\hline $\begin{array}{l}800944 \\
(65.346 .19)\end{array}$ & 40 & 45 & 50 \\
\hline
\end{tabular}

El procedimiento que se utilizó para obtener las razas del nematodo Globodera pallida (Stone) fue; un pote con llenado de suelo esterilizado a un $75 \%$, se inocularon los quistes, luego se sembró la semilla de papa y posteriormente se cubrió con una capa de suelo, cada pote tuvo una identificación de tratamiento (Figura 3).

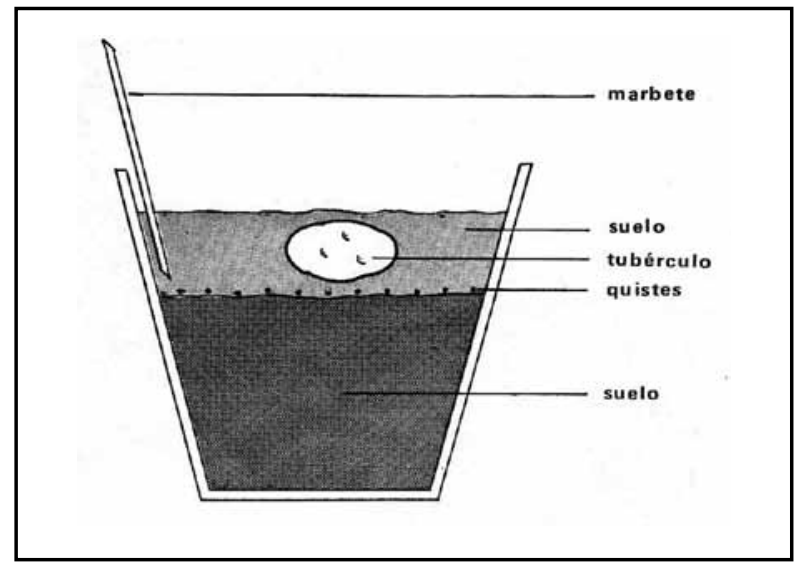

Figura 3: $\quad$ Maceta de inoculación y siembra de tubérculos. Fuente. CIP 1986. 
Canto y Scurrach en 1977 elaboraron una tabla de Esquema Latino con el fin de interpretar los resultados de razas o biotipos de Globodera pallida. Dicha tabla se utilizó en la clasificación de razas del nematodo de quiste de papa Globodera spp. La misma incluye el diferencial 800944 (65.346.19) el cual cuando es inoculado, la reacción positiva (+) determina la presencia de Globodera pallida (Stone) y la reacción negativa ( - ) presencia de Globodera rostochiensis (Canto y Scurrach 1977) (Cuadro 2).

Cuadro 2. Tabla con Esquema Latino para identificar razas de Globodera pallida. CIP. Elaborada por Canto y Scurrach 1977.

\begin{tabular}{|c|c|c|c|c|c|c|}
\hline \multirow[t]{2}{*}{ Diferenciales } & \multicolumn{6}{|c|}{ Razas de Globodera pallida (Stone) } \\
\hline & P1A & P1B & P2A P3 & $3 A$ & P4A & P5A \\
\hline $\begin{array}{l}\text { Floresta } \\
\text { (Solanum } \\
\text { tuberosum ssp } \\
\text { tuberosum) }\end{array}$ & + & + & + & + & + & + \\
\hline $\begin{array}{l}800286 \\
\text { (Multidissectum } \\
\text { híbrido P55/ 7) } \\
(\mathrm{H} 2)\end{array}$ & - & - & $+\quad+$ & + & + & + \\
\hline $\begin{array}{l}800289 \\
\text { (KTT 60.21.19) }\end{array}$ & - & - & - & - & + & + \\
\hline $\begin{array}{l}800290 \\
\text { (GLKS 58.1642.4) }\end{array}$ & + & + & + & - & + & + \\
\hline $\begin{array}{l}800291 \\
\text { (VTn 62.33.3) }\end{array}$ & - & + & - & - & - & + \\
\hline $\begin{array}{l}800944 \\
(65.346 .19)\end{array}$ & + & + & + & + & + & + \\
\hline
\end{tabular}

Al inocular, fue importante tener una población de larvas y huevos por cada quiste, por tal razón se realizó la prueba de viabilidad de los quistes. La viabilidad se hizo tomando 25 quistes y se trituraron con un homegizador o triturador de quistes (Figura 4). Luego se disolvió en un volumen de agua de $50 \mathrm{cc}$. En una pipeta se tomó 3 cc y se obtuvo el promedio de huevos y larvas por quiste, para este caso la viabilidad fue de 260 huevos y larvas por quiste. Esta técnica se hizo con el cálculo de la siguiente fórmula:

Fórmula de viabilidad de quistes

$$
\begin{gathered}
\frac{\mathrm{VT}=\text { Prom.3cc } \times \text { Vol. } \mathrm{H} 2 \mathrm{O}}{\mathrm{Q}} \\
\text { Donde: } \\
\mathrm{VT}=\text { Viabilidad Total } \\
\text { Prom= Promedio de } 3 \text { alícuotas } \\
\mathrm{Q}=\text { Número de quistes }
\end{gathered}
$$

En la Figura 4, se observa un homogenizador de quistes. La función es la de triturar los quistes para obtener las larvas y huevos.

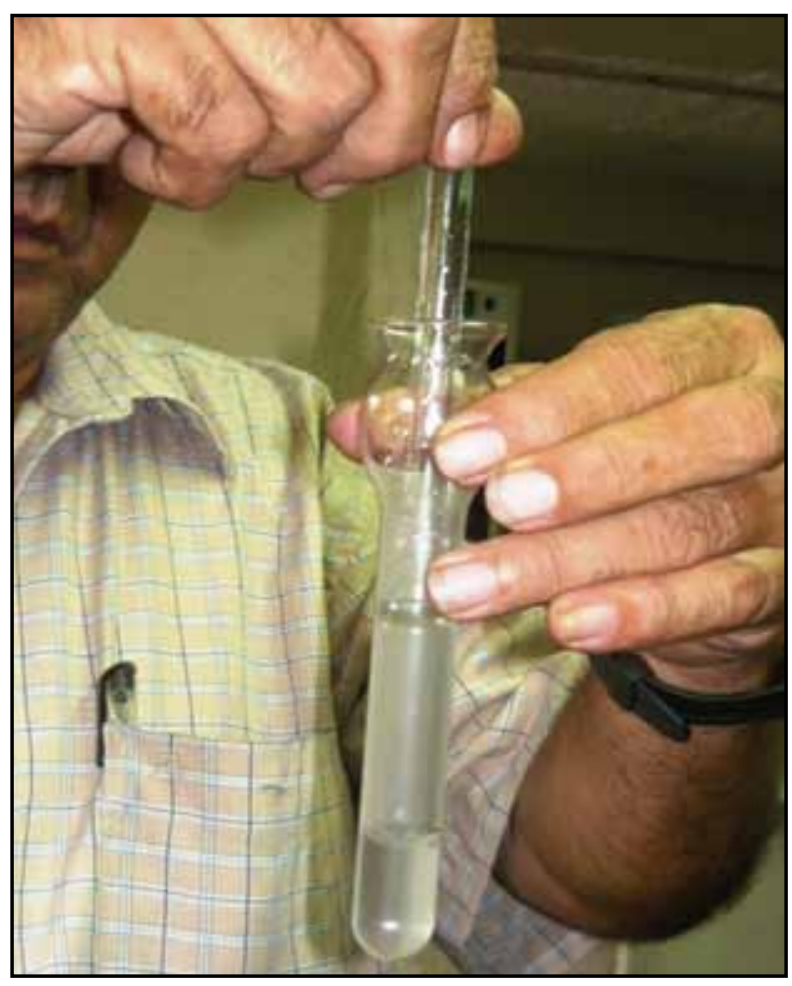

Figura 4. Homogenizador de quistes.

\section{Extracción de quistes}

\section{Método fenwick modificado}

Para poder obtener la separación de quistes de suelo, se logró con los siguientes materiales: $200 \mathrm{ml}$ de suelo seco, equipo de Fenwick. Tamiz de 100 mesh, papel filtro o papel facial, balón aforado de $250 \mathrm{ml}$ de capacidad y platos de Petri. El equipo Fenwick, consiste en un embudo colocado sobre una especie de jarra. El embudo tiene en la base en su parte 
ensanchada un tamiz de $1 \mathrm{~mm}$ de tamaño de poro. La jarra tiene forma trapezoidal, en su parte inferior presenta los soportes del embudo y una aleta inclinada que bordea la jarra como collar, pero que termina en su solo conducto. La jarra tiene su parte interior un tapón que se retira para desaguar y limpiar.

Los quistes recogidos en el tamiz de 100 mesh fueron transferidos a un balón aforado de $250 \mathrm{ml}$ y llenado hasta la mitad con agua, se agitó bien para mezclar la muestra y después se llenó el balón por completo con agua. Se dejó en reposo un minuto para que los quistes floten y el resto de materia orgánica precipite, después se vaciaron los quistes sobre un papel filtro que fue colocado previamente en el embudo de manera que mientras vaciaba estuvo rotando sobre el balón y evitando que el material orgánico se pase al filtro. Luego una vez secada la muestra se realizó la escogencia y el conteo de quistes en el estereoscopio para posterior inoculación (Figura 5).

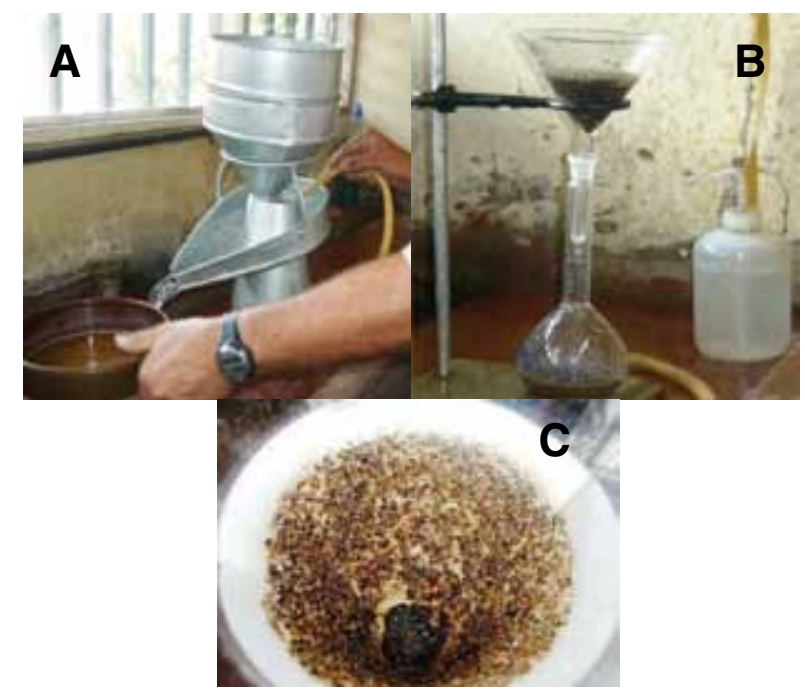

Figura 5. Fenwick Modificado (A), balón aforado de $250 \mathrm{ml}$ (B) y filtro para la extracción de quistes (C).

\section{RESULTADOS Y DISCUSIÓN}

El Cuadro 3, mostró los datos de temperatura del suelo, condición muy importante para la plaga, y este evidenció un promedio de una temperatura mínima de $9,17 \stackrel{\circ}{\circ}$ y una máxima de $30,10{ }^{\circ} \mathrm{C}$, para un promedio general de $16,92{ }^{\circ} \mathrm{C}$. Lo anterior es considerado como una condición favorable para la planta y la plaga en estudio.

Cuadro 3. Temperatura de suelo durante la investigación. San Juan de Chicuá, Cartago. Costa Rica. 2008.

\begin{tabular}{lccc}
\hline Meses & ${ }^{\circ} \mathbf{C}$ Mínima & ${ }^{\circ} \mathbf{C}$ Máxima & ${ }^{\circ} \mathbf{C}$ Promedio \\
\hline Abril & 2,30 & 29,90 & 12,37 \\
\hline Mayo & 4,50 & 29,50 & 12,48 \\
\hline Junio & 4,00 & 33,20 & 14,04 \\
\hline Julio & 19,00 & 27,60 & 24,25 \\
\hline Promedio & 9,17 & 30,10 & 16,92 \\
\hline
\end{tabular}

En el Cuadro 4 y Figura 6 se observó que los clones 800289 Solanun kurtzianum KTT $60.21 .19,800290$ Solanum vernei GLKS, 800291 Vernei VTn. no fueron infectados, lo que se manifestó como resistencia al nematodo, porque no se observó ningún quiste en las raíces de la planta, dando negativa la reacción ante la inoculación de los quistes (-).

Los clones Floresta, 800286 y 800944 se observaron como susceptibles dando una respuesta positiva (+). También, en el Cuadro 4, se determinó que el diferencial 800290 , además que resultó negativo, obtuvo el mayor peso que los demás clones. Se resaltó que la variedad floresta obtuvo el menor rendimiento en peso de papa y con quistes adheridos a la raíz. 
Cuadro 4. Resultados de peso en gramos y quistes adheridos a la raíz en diferenciales de papa. San Juan de Chicuá. Cartago. Costa Rica. 2008.

\begin{tabular}{cccc}
\hline $\begin{array}{c}\text { Diferencial } \\
\text { o clon }\end{array}$ & $\begin{array}{c}\text { Peso } \\
\mathbf{( g )}\end{array}$ & $\begin{array}{c}\text { Quistes } \\
\text { en raíz }\end{array}$ & $\begin{array}{c}\text { Reacción + o } \\
\mathbf{0}-\end{array}$ \\
\hline Floresta & 83,43 & 4,00 & + \\
800286 & 101,82 & 6,00 & + \\
800289 & 105,00 & 0,00 & - \\
800291 & 97,10 & 0,00 & - \\
800290 & 141,82 & 0,00 & - \\
800944 & 112,55 & 3,00 & + \\
\hline
\end{tabular}

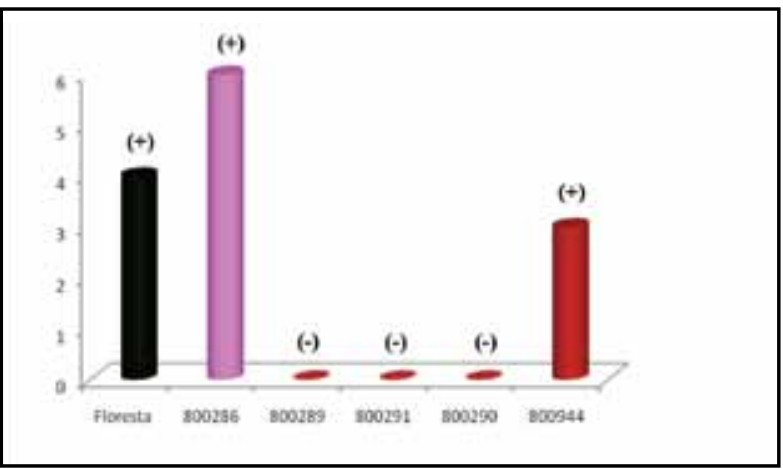

Figura 6. Efecto de las inoculaciones de quistes vrs. reproducción en raíces en cada diferencial o clon de papa. San Juan de Chicuá. Cartago, Costa Rica. 2008.

El Cuadro 5 muestra la columna del resultado de la reacción de los diferenciales y esta es idéntica a la columna donde se visualizó la raza o patotipo P3A (color rojo). Esta interpretación se basó por la metodología descrita anteriormente y sirvió para visualizar las diferentes razas de Globodera pallida (Stone). Cabe destacar que los diferenciales 800289 Solanun Kurtzianum KTT 60.21.19, 800290 Solanum vernei GLKS, 800291 vernei VTn, mostraron resistencia hacia el nematodo, porque ninguno de los clones se infectó al realizar el diagnóstico en la raíz. Lo anterior, no significa que puedan en algún momento ser susceptibles, porque se sabe que la plaga puede cambiar constantemente en su ciclo de vida, pero genéticamente la planta no, por lo tanto esta puede ser invadida por Globodera pallida (Stone) en algún momento si se siembra sucesivamente.

Cuadro 5. Resultado de clones o diferenciales inoculados con esquema latino a reacción de razas de Globodera pallida (Stone). San Juan de Chicuá. Cartago. Costa Rica. 2008

\begin{tabular}{|c|c|c|c|c|c|c|c|}
\hline \multirow{2}{*}{ Diferenciales } & \multicolumn{7}{|c|}{ Razas de Globodera pallida } \\
\hline & P1A & P1B & P2A & P3A & P4A & P5A F & Resultado \\
\hline $\begin{array}{l}\text { Floresta } \\
\text { (Solanum } \\
\text { tuberosum } \\
\text { ssp } \\
\text { tuberosum) }\end{array}$ & + & + & + & $(+)$ & + & + & $(+)$ \\
\hline $\begin{array}{l}800286 \\
\text { (Multidissectum } \\
\text { híbrido } \\
\text { P55/ 7) (H2) }\end{array}$ & - & - & + & $(+)$ & + & + & $(+)$ \\
\hline $\begin{array}{l}800289 \\
(\text { KTT } \\
60.21 .19) \\
\end{array}$ & - & - & - & $(-)$ & + & + & $(-)$ \\
\hline $\begin{array}{l}800290 \\
\text { (GLKS } \\
58.1642 .4) \\
\end{array}$ & + & + & + & $(-)$ & + & + & $(-)$ \\
\hline $\begin{array}{l}800291 \\
\text { (VTn 62.33.3) }\end{array}$ & - & + & - & $(-)$ & - & + & $(-)$ \\
\hline $\begin{array}{l}800944 \\
(65.346 .19)\end{array}$ & + & + & + & $(+)$ & + & + & $(+)$ \\
\hline
\end{tabular}

Estos resultados son importantes para trabajar con algunas líneas de tolerancia al nematodo y también con variedades que puedan excluirse para su reproducción, tomando en cuenta evitar la multiplicación por ser variedades susceptibles. Lo interesante es que tenemos clones con tolerancia o resistencia a la plaga y así se puede empezar a utilizar variedades contra la plaga en el futuro.

\section{CONCLUSIONES}

Se concluye que el clon 800286 de gen $\mathrm{H} 2$ Híbrido Multidissetum es susceptible, seguido del 800944. Este último ha sido considerado 
como susceptible a Globodera pallida (Stone), lo mismo que la variedad Floresta (S.tuberosun) De acuerdo con la metodología descrita, el ensayo presentó reacciones importantes a la raza o biotipo P3A para Globodera pallida Stone encontrada en la zona en estudio.

Las líneas o clones resistentes al nematodo son: 800289 Solanum kurtzianum KTT 60.21 .19 , 800290 Solanum vernei GLKS, 800291 Vernei VTn. Lo anterior sirve para implementar un sistema de producción integral que incluya rotación de cultivos y el uso de nematicidas químicos y biológicos.

Se debe seguir investigando y realizando pruebas de identificación de razas o patotipos debido a que la plaga se identificó en Costa Rica hace pocos años. Es posible que se encuentren más razas o patotipos en un futuro no muy lejano.

Hay que considerar que aunque se ejecute un plan de manejo, no existe la posibilidad de eliminar la plaga, debido a que los índices de multiplicación y por ende la persistencia del mismo en el suelo se debe a su constante ciclo de vida, cuando sus condiciones de clima y huésped es apropiado.

\section{LITERATURA CITADA}

Alfaro R, C. 2009. Dirección de Programas Nacionales, Programa Nacional Sectorial de papa. Disponible: gerencias-mag@mag.go.cr. www.mag.go.cr./prog-nac-papaanteceentes.html.

Brenes, A., Rivera C.; Vásquez V. 2002. Cultivo de papa en Costa Rica. San José, Costa Rica. EUNED. 2002. 120 p.

Canto, M.; Scurrach, M. 1977. Races and the pota cyst nematode in the Andean region and new system of classification. Nematologica. (23): 340-349.
Crozzoli P.; R. 1994. Temas de nematología Agrícola I. Universidad Central de Venezuela, Facultad de Agronomía, Comisión de información p. 8-9.

Franco, J.; González, A.; Matos, A. 1993. Manejo integrado del nematodo de quiste de papa. Centro Internacional de la Papa. Programa de investigación de la Papa (PROINPA) $30 \mathrm{p}$.

Greco, N. Di Vito, M. Brondonisio, A, Giordano, L, De Marins, G. 1982. The effect of Globodera pallida and G. rostochiensis on potato yield. Nematologica (28): 379-386

Greco, N. 1993. Nematodo problems affecting potato production in subtropical climate. Nematropica (23): 213-220.

Huijsman , C. A. 1960. The Influence of resistant potato varieties in soil populations Heterodera rostochiensis. Nematologica (6): 177-180. 